\title{
SATB1 enhances gastric cancer progression and metastasis via upregulation of CLIP4/DZIP1/PRICKLE2 axis in gastric cancer patients
}

\author{
SAMINA GUL ${ }^{1,2}$, AMJAD ALI ${ }^{1}$, SHAHZAD AHMAD ${ }^{3}$, CHONGYI ZHAO ${ }^{4}$, \\ ASAD ALI SHAH ${ }^{5}$, KASHIF RAHIM ${ }^{6}$, NAILA AZAM ${ }^{7}$, MOHAMMAD TARIQ ${ }^{8}$, \\ YASIR SARDAR ${ }^{9}$, FIRASAT HUSSAIN ${ }^{6}$, SARAJ BAHADUR ${ }^{10}$, MUHAMMAD \\ SHUAIB $^{11}$ \\ ${ }^{1}$ Institute of Integrative Biosciences, CECOS University of IT and Emerging Sciences, Sector F5, Phase 6, Peshawar, \\ KPK, Pakistan \\ 2 Lab of Molecular Genetics of Aging and Tumor, Faculty of Medicine, Kunming University of Science and \\ Technology, China \\ ${ }^{3}$ Institute of Basic Medical Sciences, Khyber Medical University, Peshawar, Pakistan \\ ${ }^{4}$ Faculty of Life Science and Technology, Kunming University of Science and Technology, Kunming, Yunnan, China \\ ${ }^{5}$ Medical Research Centre, Jazan University, Saudi Arabia \\ ${ }^{6}$ Department of Microbiology, Cholistan University of Veterinary \& Animal Sciences Bahawalpur 63100, Pakistan \\ ${ }_{8}^{7}$ Centre of Plant Biodiversity, University of Peshawar, Pakistan \\ 8 Institute of Experimental and Clinical Research, Pole of Endocrinology, Diabetes and Nutrition, Université \\ catholique de Louvain, Brussels, Belgium \\ ${ }^{9}$ Pak International Medical Collage, Phase 5, Hayatabad, Peshawar, Pakistan \\ ${ }^{10}$ College of Forestry, Hainan University, Haikou, China \\ ${ }^{11}$ School of Ecology and Environmental Science, Yunnan University, Kunming, China
}

\begin{abstract}
SATB1 (Special AT-rich sequence binding protein 1) plays key role in chromatin remodeling and geneexpression. SATB1 has been shown to promote invasion, migration and metastasis. However, the underlying molecular understood. Here, we show that SATB1 plays critical role in GC progression. SATB1 upregulates in GC patients' samples and shows co-apmlification with a subset of oncogenic proteins (PRICKLE2, ZEB1, CBWX7, WWTR1, ENAH, DZIP1, IGSF11, ZSCAN18, GFRA2, GFRA1, FGFR1, HDAC4, GHR, TIMP3, CLIP4, TAGLN and ILK) in different subtypes of GC. SATB1 shows positive correlation with GC promoting oncogenes and enhances the expression of metastasis associated genes in GC samples to potentiate GC progression. SATB1 enhances WNT/NOTCH signaling axis in GC samples. In contrast, STAB1 suppresses a network of tumor suppressor genes in GC samples. Moreover, SATB1 expression negatively correlates with GC patient's survival. Importantly, we found that SATB1 co-amplified genes CLIP4, DZIP1 and PRICKLE2 independently involve in GC progression. Overexpression of CLIP4, DZIP1 and PRICKLE2 show poor survival rates in GC patients. DZIP1 empowers AKT3/FGF2/FGFR1 and CXCR3/CXCR6 in GC patients. PRICKLE2 enhances GC progression by targeting FGFR1/NOTCH3/WNT4 signaling. Taken together, these results identified novel roles of SATB1, CLIP4, DZIP1 and PRICKLE2 in the GC invasion, migration and metastasis using cancer bioinformatics approach. These results highlights clinical significance of SATB1, CLIP4, DZIP1 and PRICKLE2 in GC patients and furthermore, these proteins may serve as prognostic markers in GC. These results also provide SATB1, CLIP4, DZIP1 and PRICKLE2 as potential chemotherapeutic targets in treatment of gastric cancer patients.
\end{abstract}

Keywords Gastric cancer, SATB1, PRICKLE2, DZIP1, Metastasis

To cite this article: GUL S, ALI A, AHMAD S, ZHAO C, SHAH AA, RAHIM K, AZAM N, TARIQ M, SARDAR Y, HUSSAIN F, BAHADUR S, SHUAIB M,. SATB1 enhances gastric cancer progression and metastasis via upregulation of CLIP4/DZIP1/ PRICKLE2 axis in gastric cancer patients. Rom Biotechnol Lett. 2021; 26(6): 3062-3073. DOI: 10.25083/rbl/26.6/3062-3073.

*Corresponding author: MUHAMMAD SHUAIB, School of Ecology and Environmental Science, Yunnan University, Kunming, China.

E-mail: zeyadz44@yahoo.com 


\section{Introduction}

Gastric cancer (GC) is one of the leading causes of cancer associated deaths worldwide and represents primary tumors of the digestive tract [1-3]. GC is very aggressive in nature and mostly metastasizes to intestine to produce gastrointestinal tract (GIT) cancer. Patients suffering from GC show poor prognosis and most of the patients develop secondary cancer after chemotherapy $[4$, 5]. The overall survival rates of GC patients within 5 years are approximately $15 \%$. GC arises due to genomic instability, amplification of oncogenic proteins, and mutations in tumor suppressor genes. Local recurrence and distant metastasis in GC patients is common even after surgical resection. GC patients, mostly undergo adjuvant therapy following gastric surgery to decrease recurrence [6-9]. It has been shown that metastasis and EMT related proteins and signaling pathways play important role in the progression of GC [10-12]. However, underlying complex cellular and molecular events involve in the GC development and progression still poorly understood. Moreover, analysis of gastric cancer samples at the molecular level remains unclear. Therefore, identification of novel molecular and pathogenic mechanisms that promote GC progression in GC patients need to be addressed. Importantly, the development of independent and potential prognostic biomarkers is urgently required for the diagnosis and effective treatment of $\mathrm{GC}$ patients.

SATB1 is a nuclear matrix associated protein that regulates chromatin remodeling and controls gene expression at the chromatin level $[13,14]$. SATB1 has been shown to promote cancer metastasis, invasion and migration. Overexpression of SATB1 is associated with tumor development. In contrast, knockdown of SATB1 in cancer cells decreases proliferation, invasion and migration [15-18]. It has been shown that SATB1 enhance $\mathrm{C}-\mathrm{MYC}$ and $\beta$-Catenin expression to promote colorectal cancer $[19,20]$. Recent studies showed that SATB1 expression is inhibited by MicroRNA-495 and MicroRNA-23a in renal cancer and osteosarcoma respectively [21, 22]. SATB1 also promote neurogenesis and play important role in immune tolerance [23]. SATB1 has been shown to enhance BCL2 transcription to inhibit apoptosis [24]. SATB1 expression has been shown to overexpress in prostate cancer, breast cancer, colorectal cancer, renal carcinoma, squamous carcinoma, bladder cancer, liver cancer and glioma. It is well established that SATB1 play critical role in the cancer pathogenesis by targeting metastasis and epithelial-mesenchymal-transition (EMT). Clinical studies on SATB1 revealed that, SATB1 is an independent prognostic marker in several cancers, including breast, colorectal and prostate cancer [26-28]. However, the role of SATB1 in gastric cancer progression and development remain poorly understood. CLIP4 is CAP-GLY domain containing linker protein 4 and also known as restin like protein $2[29,30]$. The role of CLIP4 in cancer progression remains unknown. DZIP1 is a zinc finger protein, which play an important role in cilium formation. DZIP1 mainly express in germ cells and embryonic stem cells. Function of DZIP1 in cancer development remains unclear [31, 32]. PRICKLE2 (Prickle homolog 2) is a nuclear translocation receptor and have an important role in the movement of nerve cells [33]. However, the function of PRICKLE2 in cancer development remains elusive. Here, we show that SATB1, CLIP4, DZIP1 and PRICKLE2 play important roles in the progression of GC. These proteins promote GC by targeting subsets of genes that support cell growth, proliferation, invasion and metastasis. In contrast, we found that SATB1, CLIP4, DZIP1 and PRICKLE2 suppress the mRNA levels of tumor suppressor proteins. Overexpression of these proteins showpoor survival rates in $\mathrm{GC}$ patients.

\section{Materials and Methods}

\section{Analysis of CBioportal database}

In this study, we utilized publicly available cancer genomic database CBioportal (http://www.cbioportal.org/index.do), that contain a rich profile of cancer patient samples, clinical studies and integrative analysis of cancer genomics [35, 36]. CBioportal having molecular profiling data of several genes. That obtained from various cell lines and cancer tissues. We chose The Cancer Genome Atlas (TCGA) gastric cancer data sets [34] from CBioportal to perform our analysis. We query PRICKLE2, ZEB1, CBWX7, WWTR1, ENAH, DZIP1, IGSF11, ZSCAN18, GFRA2, GFRA1, FGFR1, HDAC4, GHR, TIMP3, CLIP4, TAGLN, ILK, AKT3, FGF2, FGFR1, CXCR3, CXCR6, ATF4, ATG12, BAG3 and PINK1 genes on CBioportal using the TCGA gastric cancer database. Oncoprint analysis were generated using CBioportal. SATB1 gene was selected on the basis of its upregulation in GC samples. Furthermore, we selected CLIP4, DZIP1 and PRICKLE2 genes on the basis, having positive correlation with SATB1 in GC samples using the TCGA gastric cancer database from CBioportal

\section{Analysis of Oncomine database}

Oncomine database (https:/www.oncomine.org/) is publicly available cancer genomic database that contain several types of cancer datasets including gastric cancer datasets [37, 38]. SATB1, CLIP4, DZIP1 mRNA expressions in cancer tissue samples were compared with normal tissues that was obtained using various parameters such as fold change $>2$ was used, $p$-value $<1 \mathrm{E}-4$ was selected, and the gene ranking in top $10 \%$ was selected.

\section{Gene's expression analysis.}

GC samples datasets obtained from (http:/www.cbioportal.org /index.do) were analyzed for genes expression analysis. Scatter plots were drawn using Car package that contains a scatter plot function (http://cran.r47project.or g). For box plots; box boundaries show 25 th and 75 th percentile; whisker shows 10th and 90th percentile and the line represent the median value. Linear regression analysis was used after $\log 2$ transformation.

\section{Survival Analysis}

GC samples data sets obtained from Kaplan Mier (http://www.kmplot.com/) were analyzed for Kaplan-Meier survival curves using KM-plotter. GC Patients' samples were 
divided into two groups; showing low and high 55 levels of SATB1, CLIP4, DZIP1 and PRICKLE2. High level SATB1, CLIP4, DZIP1 and PRICKLE2 groups were defined as the gastric cancer patient samples having increased levels of SATB1, CLIP4, DZIP1 and PRICKLE2 than the median, and low levels SATB1, CLIP4, DZIP1 and PRICKLE2 were defined as the gastric cancer patients having decreased levels of SATB1, CLIP4, DZIP1 and PRICKLE2 than the median.

\section{Statistical analysis}

Survival and genes expressions analysis of cancer samples were performed using R package and Bio conductor (https://cran.r-project.org/). All data results were considered statistically significant at $\mathrm{P}<0.05$.

\section{Results and discussion}

SATB1 expression upregulates in gastric cancer and correlates with clinicopathalogical features of gastric cancer patients

To explore the potential role of SATB1 in gastric cancer progression, we analyzed The Cancer Genome Atlas (TCGA) gastric cancer dataset obtained from cBioPortal for cancer genomics [34]. A total of 295 gastric cancers patient samples were examined in this dataset. We observed upregulation of SATB1 in 9 gastric samples (Figure 1A). Importantly, we observed coupregulation of SATB1 with several oncogenes such as PRICKLE2,ZEB1,CBWX7,

WWTR1,ENAH,DZIP1,IGSF11,ZSCAN18,GFRA2,GFR A1,FGFR1,HDAC4,GHR,TIMP3,CLIP4,TAGLN and ILK in gastric cancer patient samples (Figure 1A). Furthermore, we analyzed the expression of SATB1 in different subtypes of gastric cancer. We detected overexpression of SATB1 in CIN subtype of gastric cancer and intriguingly observed positive correlation of SATB1 with a subset of oncogenes in CIN subtype (Figure 1B). Importantly, we observed no co-upregulation of SATB1 with a subset of oncogenes in an EBV subtype of gastric cancer (Figure 1C). Next, we found a strong association of SATB1 with a subset of oncogenes in a GS subtype of gastric cancer samples (Figure 1D). Finally, we detected weak correlation of SATB1 with a group of oncogenic proteins in MSI subtype of gastric cancer (Figure 1E). Results from Oncomine database showed that SATB1 strongly overexpress in diffuse, intestinal and mixed gastric carcinoma to drive the gastric cancer progression (Figure 1F). Taken together, these results indicate that SATB1 strongly involve in gastric cancer development and also upregulate the expression of a subset of oncogenes to drive the gastric cancer progression in gastric cancer patients.

$\quad$ A
SATB1
PRICKLE2
ZEB1
CBB7
WWWT1
ENAH
DZIP1
IGSF11
ZSCAN18
GFRA2
GFRA1
FGFR1
HDAC4
GHR
TIMP3
CUP4
TAGLN
ILK

$\quad$ D
SATB1
PRICKLE2
ZEB1
CBX7
WWTR1
ENAH
DZIP1
IGS111
ZSCAN18
GFRA2
GFRA1
FGFR1
HDAC4
GHR
TIMP3
CUP4
TAGLN
ILK
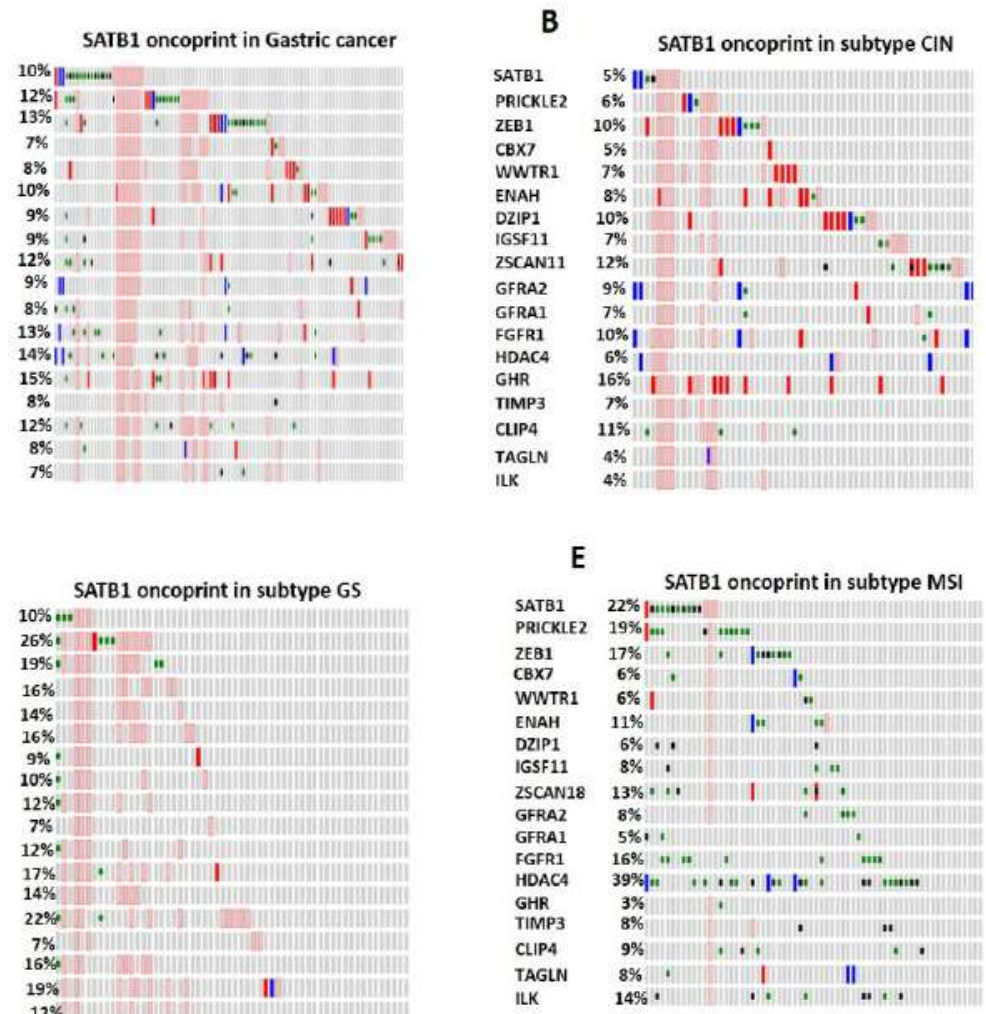
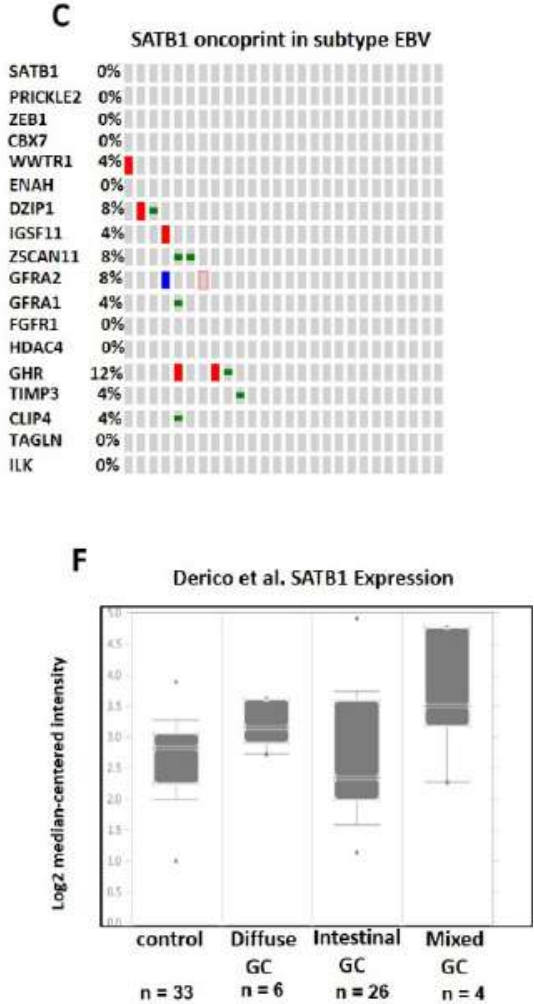

Figure 1. SATB1 expression is enhanced in GC patients' samples.

(A-E) Oncoprint analysis of SATB1 and its co-expressed genes in different subtypes of GC samples from cBio Portal cancer genomics data base (http://www.cbioportal.org/index.do) [34]. (F)Oncomine database (http://www.oncomine.com) was used to analyze the SATB1 expression in Derico et al, GC data set. Box plots showing upregulation of SATB1 in GC patients' samples. Whisker shows $10^{\text {th }}$ and $90^{\text {th }}$ percentile; line represent the median value; box boundaries show $25^{\text {th }}$ and $75^{\text {th }}$ percentile. Range of data isrepresented by dots. 
SATB1 shows positive correlation with gastric cancer promoted oncogenic proteins in gastric cancer patient samples

To further explore the potential role of SATB1 in gastric cancer progression, we selected a group of oncogenes (ENAH, HDAC4, ZEB1, KIF1B, MINA, TAGLN, ZSCAN18) that are involved in the gastric cancer progression and metastasis. We assessed the correlation of SATB1 with these oncogenic proteins in the gastric cancer TCGA dataset [34]. Importantly, we found a strong positive correlation of SATB1 with these oncogenes in gastric cancer samples (Figures 2A-2H). These results suggest that SATB1 cooperates with oncogenic proteins to further promote gastric cancer progression and metastasis. Furthermore, these results also indicate a positive feedback loop of SATB1 with oncogenic proteins in the gastric cancer progression.

SATB1 enhances the expression of metastasis associated genes in gastric cancer samples

Next, we investigated the effect of SATB1 on mRNA expression of growth promoting genes, metastasis associated genes and oncogenic signaling pathway proteins. We selected ADAM8, CD55, CD24 and CD90, which play critical roles in growth of multiple cancers. We divided gastric cancer samples into two groups showing high and low levels of SATB1 on the basis of median value.We analyzed the mRNA expression of ADAM8, CD55, CD24 and CD90 in STAB1 low and high expressing groups and found that high expression of SATB1 upregulated the expression of ADAM8, CD55, CD24 and CD90 in gastric cancer samples (Figures 3A-3D). These results indicate that SATB1 enhances the expression of growth promoting genes to potentiate gastric cancer cells growth.

To further explore the role of SATB1 in gastric cancer metastasis, we selected a subset of metastasis associated genes that promote cancer metastasis, such as STARD3, DDX27, CDK1, CDK5, HOXC6, HOXD8 and HOXD1. We analyzed the mRNA expression levels of these genes in STAB1 low and high expressing gastric cancer samples. Box plot analysis revealed that STARD3, DDX27, CDK1, CDK5, HOXC6 expression were strongly upregulated in SATB1 high expressing gastric cancer samples (Figures 3E-3K).

These results prompted us to analyze the effect of SATB1 on WNT/NOTCH axis in gastric cancer samples. WNT/NOTCH axis play a crucial role in several cancer progression including gastric cancer. Amplification and upregulation of WNT/NOTCH signaling pathway proteins is associated with aggressive gastric cancer progression, EMT and metastasis. Therefore, we analyzed the expression of WNT4, NOTCH3 and NOTCH4 signaling protein expression in STAB1 low and high expressing gastric cancer samples. Importantly, we found that high expression of SATB1 promoted the expression of WNT4, NOTCH3 and NOTCH4 in gastric cancer samples (Figures 3L-3N).
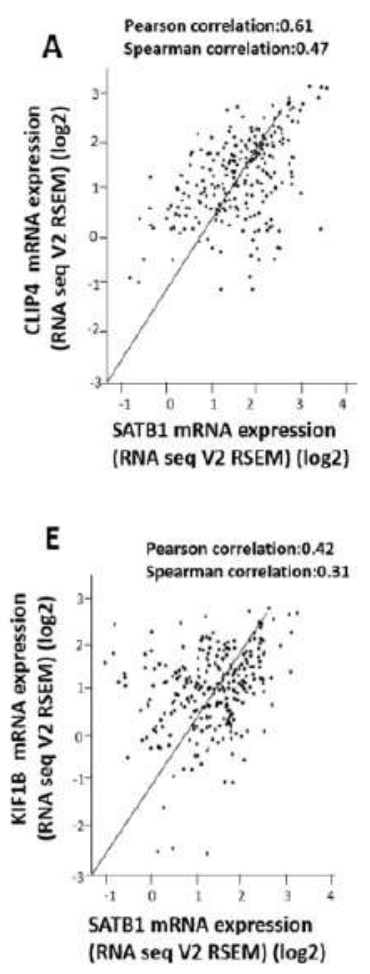
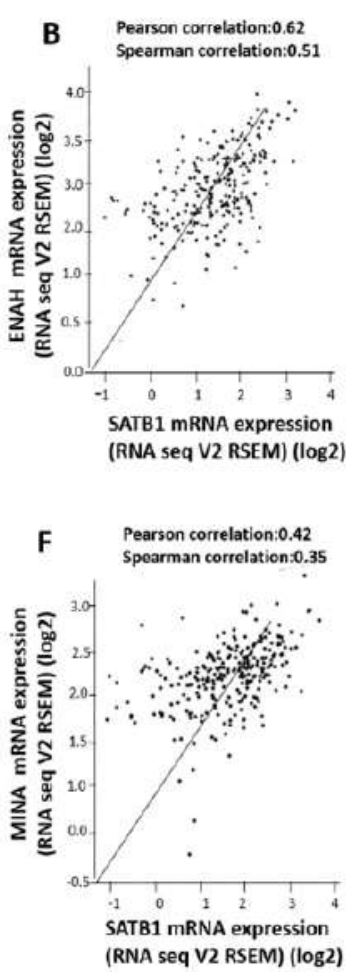
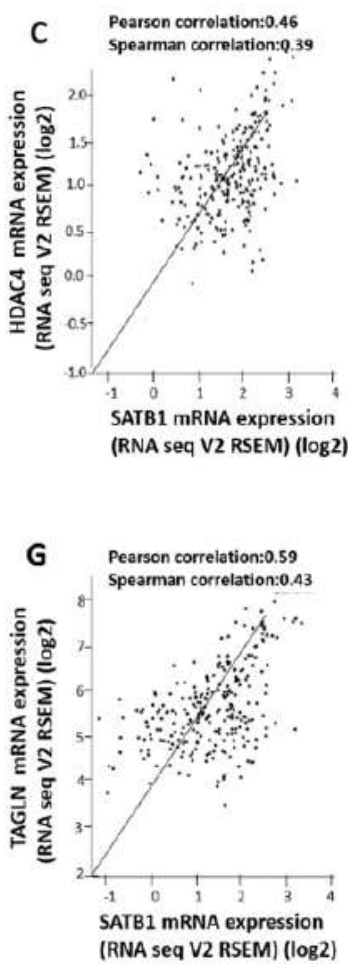
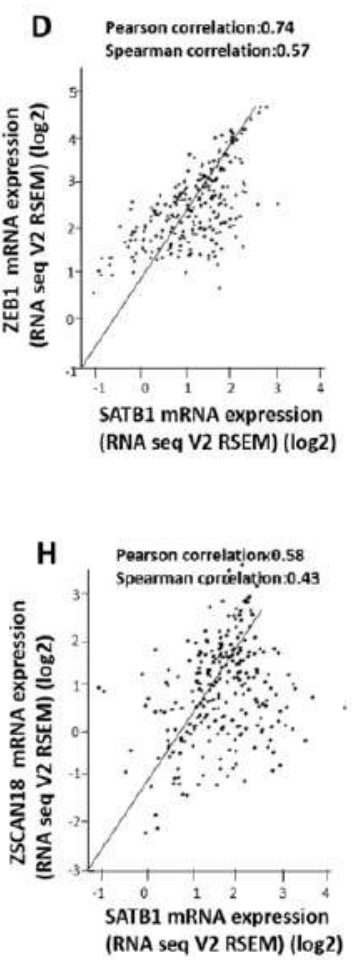

Figure 2. SATB1 shows positive correlation with several oncogenic proteins in GC samples (A-H) Positive correlation of SATB1 with cancer promoting genes. Analysis of GC samples data set from cBioPortal cancer genomics data base (http://www.cbioportal.org/index.do) [34]. Plots were drawn using Car package that contains a scatter plot function (http://cran.r-project.org). Linear regression analysis was used after $\log 2$ transformation between SATB1 and the indicated genes. 
A

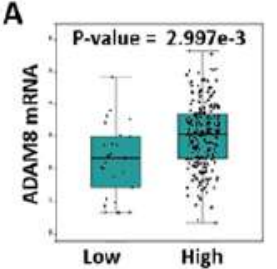

$\mathbf{F}$

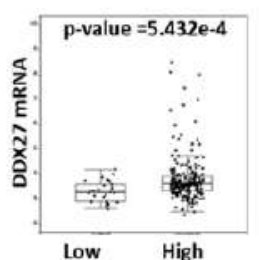

K

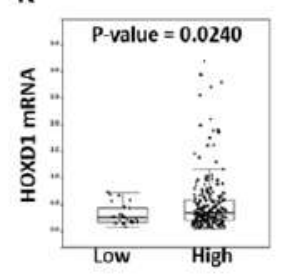

B

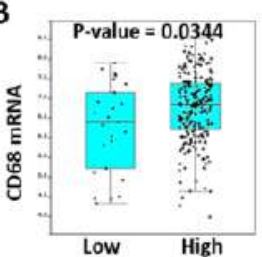

G

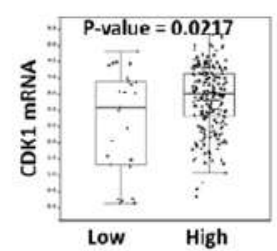

L

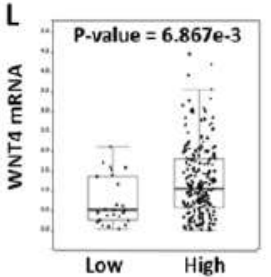

C

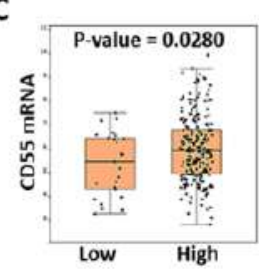

H
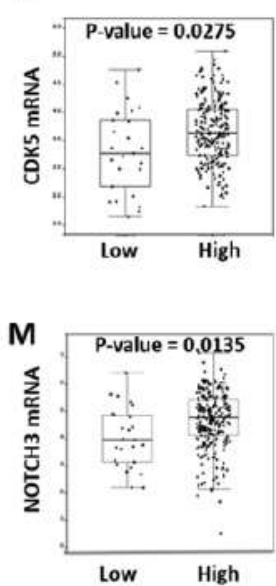

D

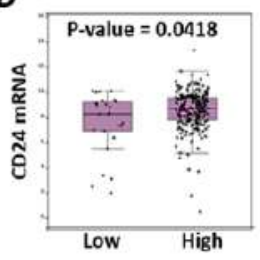

I
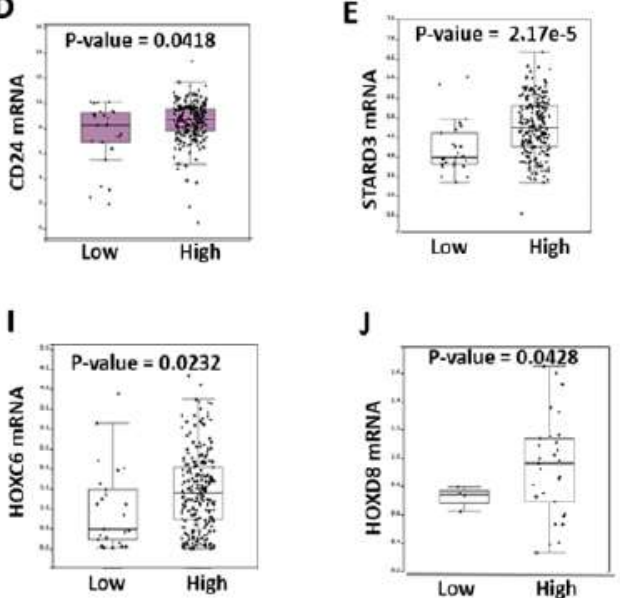

N

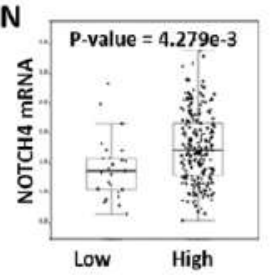

Figure 3. SATB1 induces mRNA expressions of tumor promoting genes in $\mathrm{GC}$ samples.

(A-N) GC cancer dataset from (http://www.cbioportal.org/index.do) was divided into two groups having a high and low expression of SATB1. Analysis of several oncogenic proteins showed SATB1 induces tumor promoting genes mRNA expressions in GC samples. Box plots showing changes in mRNA expression in GC samples having a high and low expression of SATB1. Box boundaries show $25^{\text {th }}$ and $75^{\text {th }}$ percentile; whisker shows $10^{\text {th }}$ and $90^{\text {th }}$ percentile; and the line represents the median value.

SATB1 inhibits tumor suppressor and autophagy promoting genes expression in gastric cancer samples

To further understand the underlying molecular mechanisms that how SATB1 enhances gastric cancer progression, we analyzed the expression of tumor suppressor genes in SATB1 high and low expression gastric cancer samples. Tumor suppressor proteins such as KLF10, CDKN1A, FOXO1, KLF12, CD81 and PTEN play key role in inhibition of cancer progression. Increased expression of these proteins suppresses cancer cell growth and progression. Analysis of mRNA expression of these tumor suppressor genes in SATB1 high and low levels groups revealed that SATB1 suppressed expression of KLF10, CDKN1A, FOXO1, KLF12, CD81 and PTEN in gastric cancer samples to promote gastric cancer progression (Figures 4A-4F). These results suggest that SATB1 empowers gastric cancer progression by downregulation of tumor suppressor genes expression.

Autophagy play important role in cancer development. Induction of autophagy suppresses cancer progression, while, inhibition of autophagy is associated with cancer cell growth, EMT, invasion, migration and metastasis. Autophagy is regulated by several autophagy related genes such as ATG12, ATF4, EEF2, BAG3 and PINK1.
These genes promote autophgy process to suppress cancer cells invasion and migration. Box plot analysis showed that expression levels of these autophagy promoting genes were downregulated in gastric cancer samples, expressing high levels of SATB1 (Figures 4G-4K). Thus, SATB1 inhibits autophagy to promote invasion, migration and metastasis in gastric cancer patients. Importantly, these results indicate that SATB1 target several signaling pathways and proteins in the gastric cancer progression.

\section{SATB1 overexpression shows poor survival in gastric} cancer patient samples

To further explore the relationship between SATB1levels and gastric cancer patient survival, we analyzed publicly available gastric cancer datasets from the Kaplan-Mier survival curves database. In each gastric cancer dataset, we divided gastric cancer patients into two groups based on SATB1 expression. KM plot showed that gastric cancer patients expressing high levels of SATB1 show poor survival (Figures 5A-5G). In contrast, gastric cancer patients having low expression of SATB1 showed better survival in multiple gastric cancer data sets(Figures $5 \mathrm{~A}-5 \mathrm{G})$. Taken together, these results indicate that high expression of SATB1 drive gastric cancer progression, which correlates with poor survival of gastric cancer patients. 


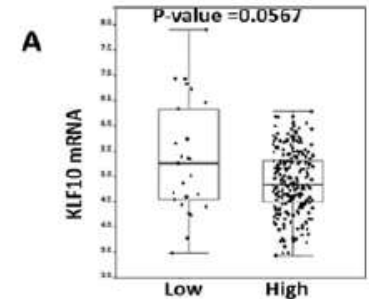

E
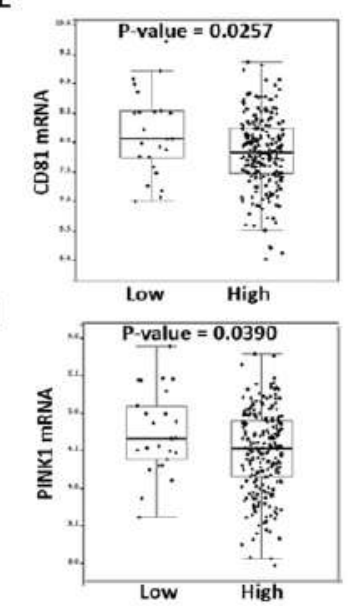

B

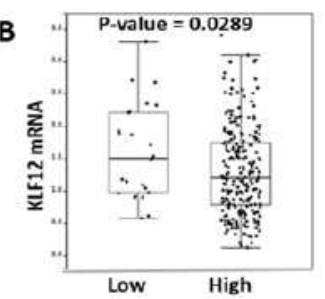

$\mathbf{F}$

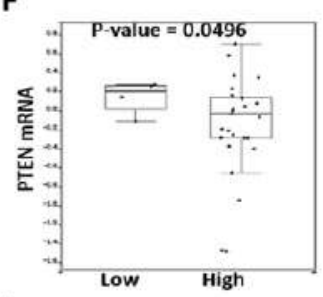

J

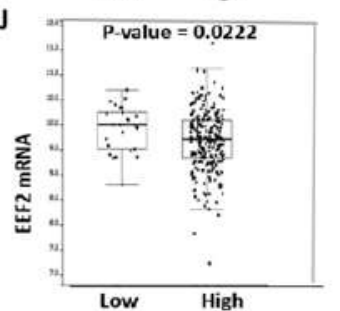

C

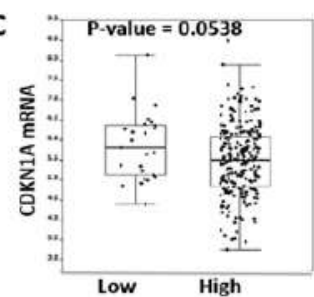

G
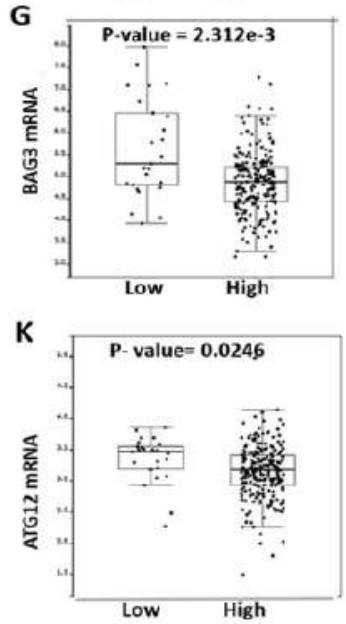

D

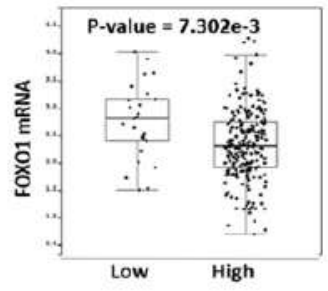

H

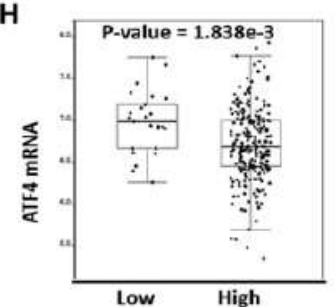

Figure 4. High expression of SATB1 reduces tumor suppressor genes expressions in GC samples.

(A-K) GC cancer dataset from (http://www.cbioportal.org/index.do) was divided into two groups having a high and low expression ofSATB1. Analysis of several tumor suppressor proteins showed SATB1 inhibits tumor suppressor genes mRNA expressions in GC samples. Box plots showing changes in mRNA expression in GC samples having a high and low expression of SATB1. Box boundaries show $25^{\text {th }}$ and $75^{\text {th }}$ percentile; whisker shows $10^{\text {th }}$ and $90^{\text {th }}$ percentile; and the line represents the median value.
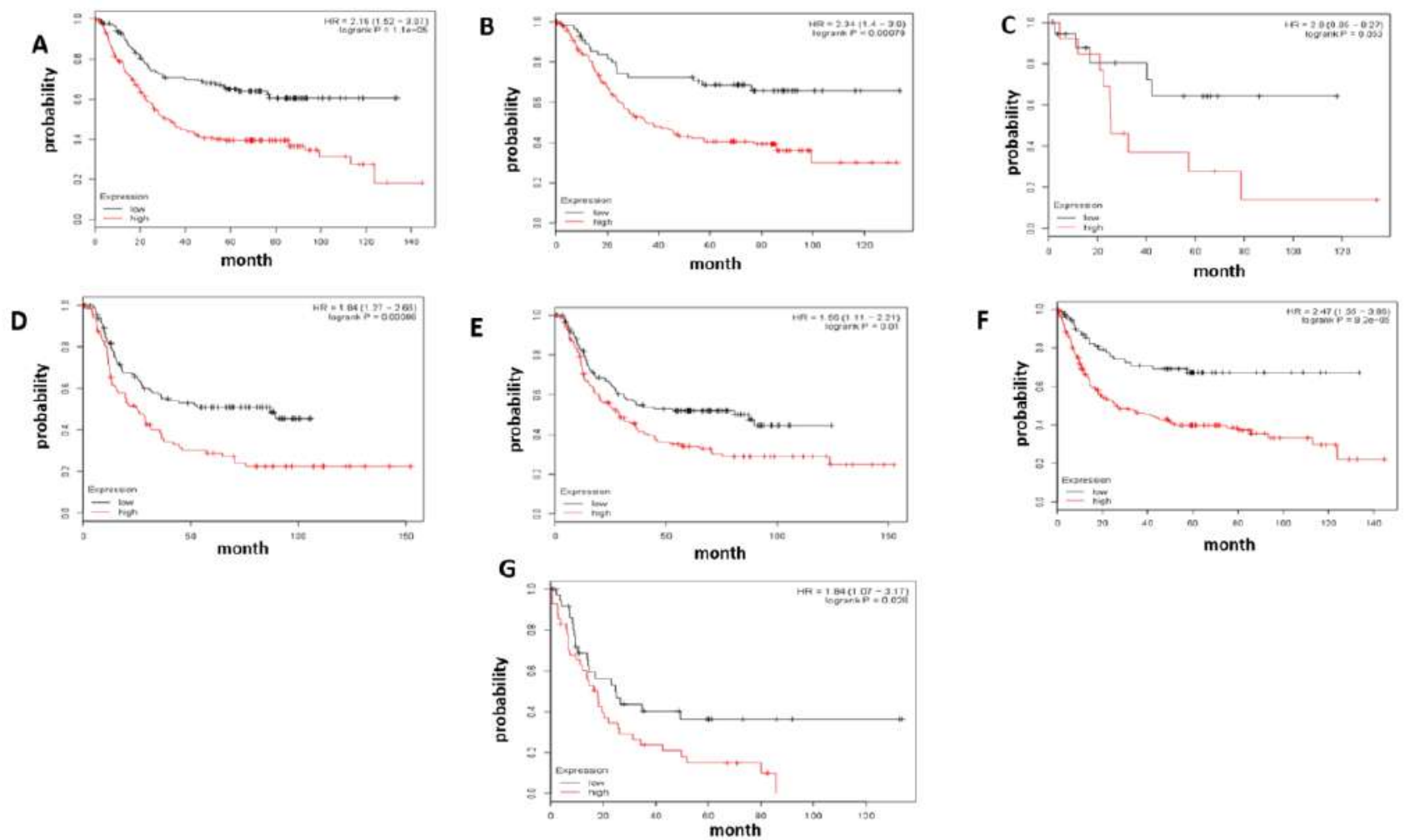

Figure 5. High SATB1 expression correlates with poor survival of GC patients

(A-G) Survival curves fromKM plotter (http://www.kmplot.com/) using GC samples showing that GC patients having levels of STAB1 show poor survival. The GC samples were divided at the median value into two groups having a high and low expression of SATB1 
CLIP4 expression enhances gastric cancer progression and correlates with clinic-pathological features of gastric cancer patients

Results from the oncoprint analysis in figure 1, showed that CLIP4 co-express with SATB1 in gastric cancer samples. Therefore, these hints prompted us to investigate the independent effect of CLIP4 on gastric cancer progression. We divided gastric cancer samples on the basis of CLIP4 expression into two groups. Scatter plot analysis showed that CLIP expression was positively correlated with STARD8 and ZEB1 levels in gastric cancer samples (Figures 6A and 6B). Furthermore, box plot analysis revealed that ADAM8, STARD3, and MMP11expressions were strongly upregulated in gastric cancer samples expressing high levels of CLIP4 (Figures 6C-6E). In contrast, we observed significant downregulation in BAG3, PINK1 and CDKN1A tumor suppressor genes expression in gastric cancer samples showing high levels of CLIP4(Figures 6F-6H).

Next, we investigated the relationship between CLIP4 and gastric cancer patient survival using KM plotter. We analyzed a gastric cancer dataset and found that high CLIP4 expression was associated with poor survival rates of gastric cancer patients (Figure 6I). Importantly, results from oncomine database showed that CLIP4 expression enhanced in diffuse, mixed and intestinal gastric carcinoma (Figure 6J). Furthermore, datasets from oncomine database showed that CLIP4 overexpress in gastric cancer and metastasis (Figures $6 \mathrm{~K}$ and $6 \mathrm{~L}$ ). Taken together, these results indicate that CLIP4 play key role in gastric cancer progression, invasion and metastasis by targeting a subset of gene network.
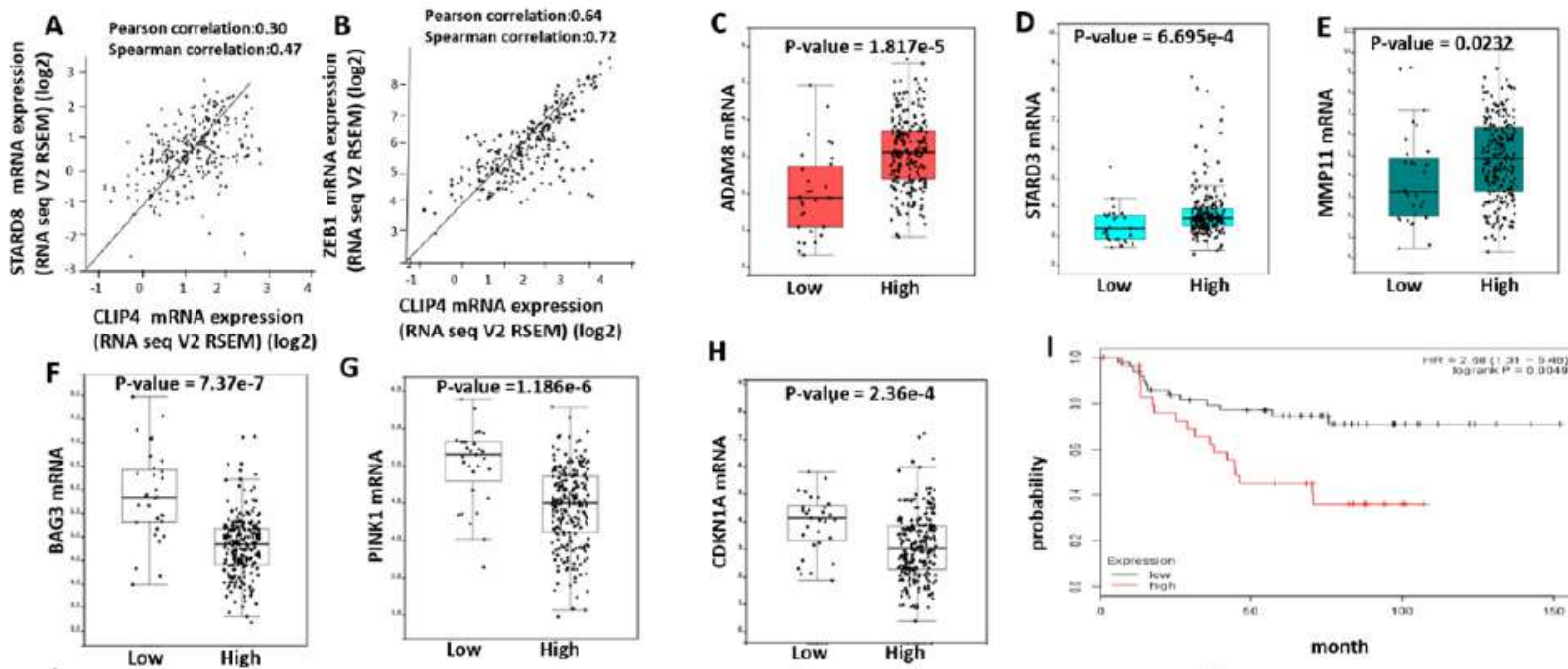

G

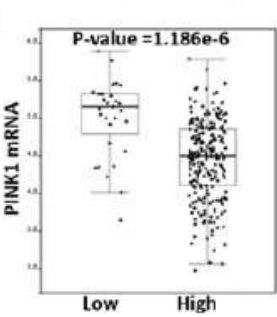

H
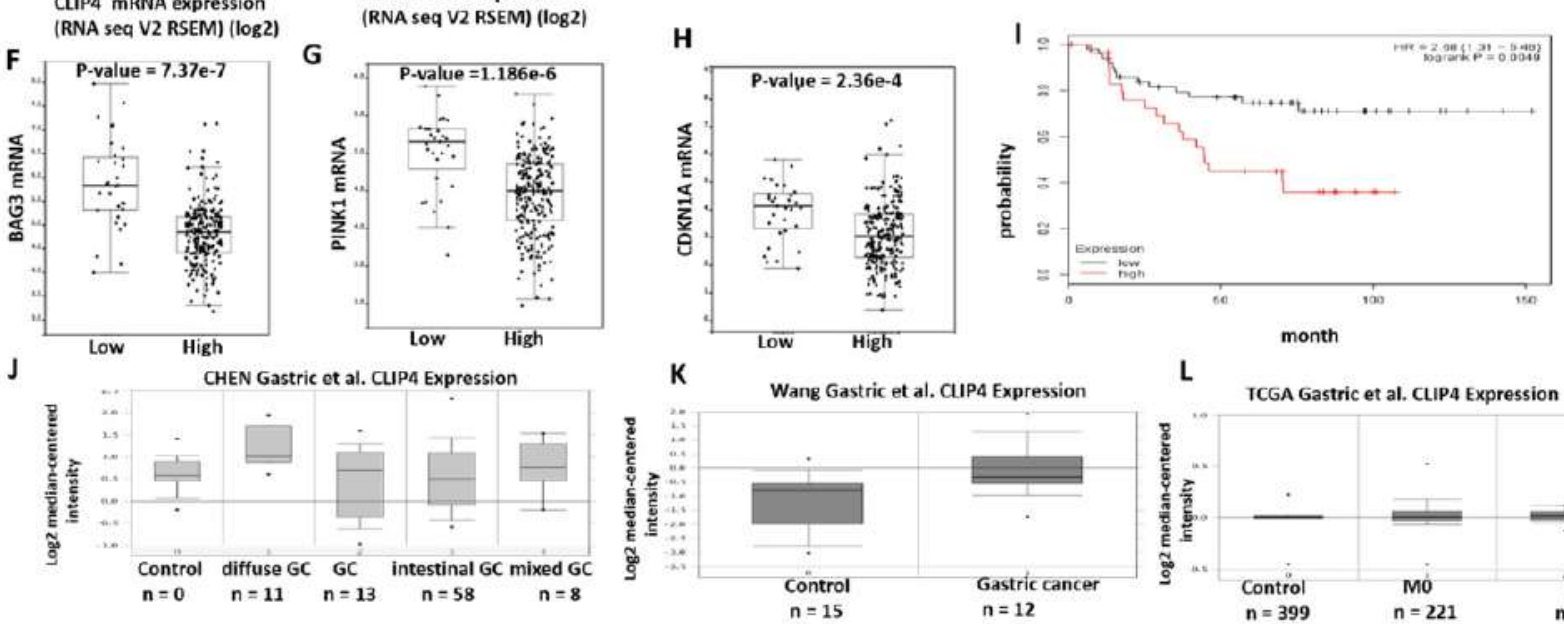

L

TCGA Gastric et al. CUIP4 Expression

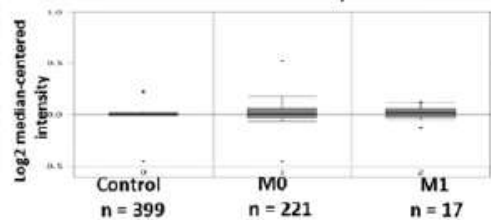

Figure 6. CLIP4 promotes gastric cancer progression

(A and B) Positive correlation of CLIP4 with STARD8 and ZEB1. Analysis of GC samples data set from cBioPortal cancer genomics database (http://www.cbioportal.org/index.do). Plots were drawn using Car package that contains a scatter plot function (http://cran.r-project.org). Linear regression analysis was used after $\log 2$ transformation betweenCLIP4 and STARD8; andbetween CLIP4 and ZEB1.

(C-H) GC cancer dataset from (http://www.cbioportal.org/index.do) was divided into two groups having a high and low expression of CLIP4. (C-E) Analysis of several oncogenic proteins showed CLIP4 enhances tumor Promoting genes mRNA expressions in GC samples.

(F-H) Analysis of several tumor suppressor proteins 59 showed CLIP4 inhibits tumor suppressor genes mRNA expressions in GC samples. Box plots showing changes in mRNA expression in GC samples having a high and low expression of CLIP4. Box boundaries show $25^{\text {th }}$ and $75^{\text {th }}$ percentile; whisker shows $10^{\text {th }}$ and $90^{\text {th }}$ percentile; and the line represents the median value.

(I) Survival curves fromKM plotter (http://www.kmplot.com/) using GC samples showing that GC patients having levels of CLIP4 show poor survival. The GC samples were divided at the median value into two groups having high and low expression of CLIP4.

(J-L) Oncomine database (http://www.oncomine.com) was used to analyze the CLIP4 expression in GC samples Data percentile; sets. Box line plots represent showing the upregulation median value of; box CLIP4 boundaries in GC show patients' $25_{\text {th }}$ samples .and $75_{\text {th }}$ Whisker percentile shows. Range $10^{\text {th }}$ of and data $90^{\text {th }}$ represented by dots. 


\section{DZIP1 enhances gastric cancer progression via upregulation of AKT3/FGF2/FGFR1 and CXCR3/CXCR6 axis}

Since our results indicate that DZIP1 show positive correlation with SATB1 in gastric cancer samples, therefore we aimed to investigate independent role of DZIP1 in gastric cancer progression. We divided gastric cancer samples into two groups on the basis of DZIP1 expression. Scatter plot analysis showed that CLIP4 expression was strongly correlated with AKT3, FGF2 and FGFR1 expression in gastric cancer samples (Figures 7A-7C). AKT3, FGF2 and FGFR1 play crucial roles in gastric cancer progression. Positive correlation of these oncogenic proteins with DZIP1 further promotes gastric cancer cells invasion and migration. Moreover, box plot analysis showed that CXCR3 and CXR6 expressions were strongly upregulated in DZIP1 high expressing gastric cancer samples (Figures 7D and 7E). These results indicate that
DZIP1 enhances CXCR3/CXCR6 axis to potentiate gastric cancer progression. Moreover, GC samples having an increased expression of DZIP1 showed decreased levels of tumor suppressor and autophagy promoting genes expression (Figures7F-7I).

Next, we analyzed the correlation of DZIP1 with gastric cancer patient survival by KM plotter and found that gastric cancer patients expressing high levels of DZIP1 show poor survival rates in comparison to gastric cancer patients having low expression of DZIP1(Figures $7 \mathrm{~J}$ and $7 \mathrm{~K}$ ). Furthermore, results from oncomine database revealed that DZIP1 expression was significantly upregualted in gastric cancer patients (Figure 7L). Taken together, these results suggest that DZIP1 amplification occurs in gastric cancer patients. DZIP promotes the expression of growth related protein to further enhance gastric cancer invasion and metastasis.
A

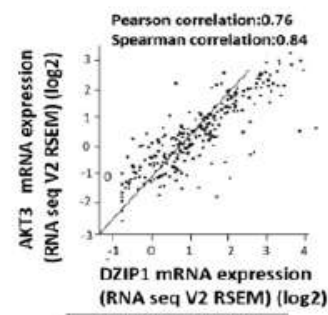

E

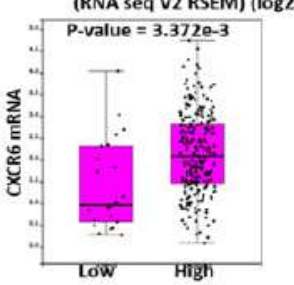

I

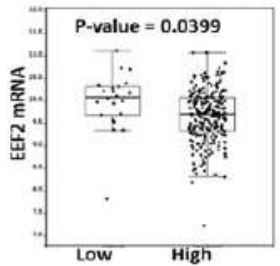

B

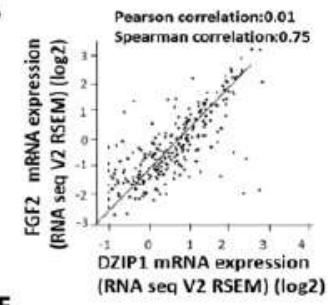

F

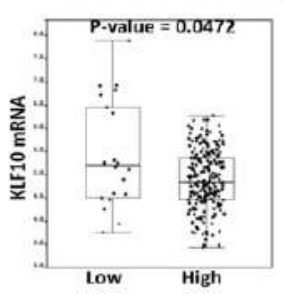

J

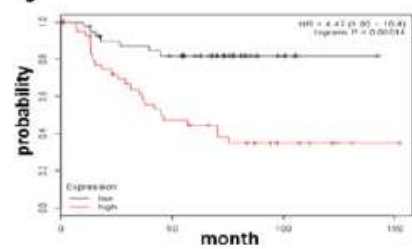

C

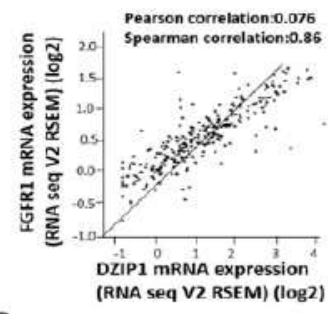

G

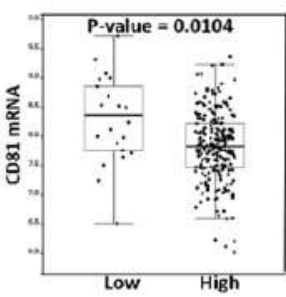

K

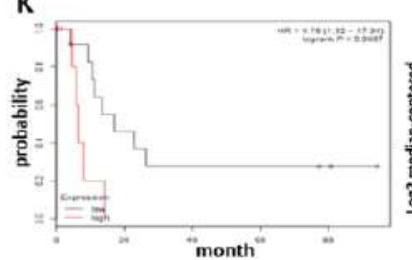

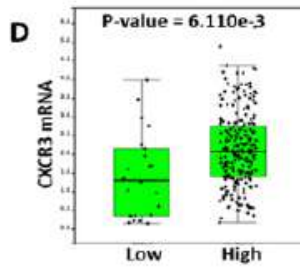

H

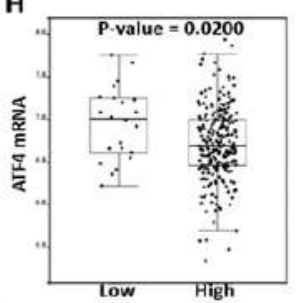

L Wang Gastric et al. DZIP1 Expression

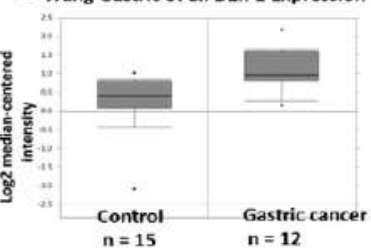

Figure 7. DZIP1 is involved in the gastric cancer progression.

(A-C) Positive correlation of DZIP1 with AKT3, FGF2 and FGFR1. Analysis of GC samples data set from cBioPortal cancer genomics database (http://www.cbioportal.org/index.do). Plots were drawn using Car package that contains a scatter plot function (http://cran.r-project.org). Linear regression analysis was used after $\log 2$ transformation betweenDZIP1andAKT3; between DZIP1 and FGF2; and between DZIP1 and FGFR1.

(D-I) GC cancer dataset from (http://www.cbioportal.org/index.do) was divided into two groups having a high and low expression ofDZIP1.

(D and E) Analysis of CXCR3 and CXCR6 expression showed DZIP1 enhances tumor these genes expressions in GC samples. (F-I) Analysis of several tumor suppressor proteins showed DZIP1inhibits tumor suppressor genes mRNA expressions in GC samples. Box plots showing changes in mRNA expression in GC samples havinga high and low expression of DZIP. Box boundaries show $25^{\text {th }}$ and $75^{\text {th }}$ percentile; whisker shows $10^{\text {th }}$ and $90^{\text {th }}$ percentile; and the line represents the median value.

(J and K) Survival curves fromKM plotter (http://www.kmplot.com/) using GC samples showing that GC patients having levels of DZIP1show poor survival. The GC samples were divided at the median value into two groups havinga high and low expression of DZIP1.

(L) Oncomine database (http://www.oncomine.com) was used to analyze the DZIP1 expression in GC samples dataset. Box plots showing upregulation of DZIP1 in GC patients' samples. Whisker shows $10^{\text {th }}$ and $90^{\text {th }}$ percentile; line represents the median value; box boundaries show $25^{\text {th }}$ and $75^{\text {th }}$ percentile. Range of data is represented by dots. 
PRICKLE2 enhances gastric cancer progression by targeting FGFR1/NOTCH3/WNT4 signaling

We observed positive correlation of SATB1 with PRICKLE2 in gastric cancer samples, there we sought to further investigate the role of PRICKL2 in the gastric cancer invasion. Scatter plot analysis showed positive correlation of PRICKLE2 with with FGFR1 and ZEB1(Figures 8A and 8B). FGFR1 promote gastric cancer cells growth and invasion, while, ZEB promote gastric cancer EMT. These results suggest that PRICKLE2 promote gastric cancer invasion, migration and EMT like features by targeting FGFR1 and ZEB1.

Next, we observed that STARD3, NOTCH3 and WNT4 mRNA levels were significantly induced in gastric cancer samples that express high levels of PRICKLE2 (Figures 8C-8E). NOTCH and WNT signaling pathways play crucial role in gastric cancer metastasis. Positive correlation of PRICKLE2 with NOTCH3 and WNT4 signaling molecules indicates that PRICKLE2 may potentiate NOTCH/WNT axis to promote gastric cancer invasion and metastasis.

Importantly, we observed decreased mRNA expression of KLF10, FOXO1, ATG12 and ATF4 tumor suppressor genes in gastric cancer samples that having high levels of PRICKLE2 (Figures 8F-8I). Thus, PRICKLE2 show negative correlation with tumor suppressor genes to promote gastric cancer. KM plotter showed that gastric cancer patients expressing high levels of PRICKL2 showed decreased survival rates (Figures $8 \mathrm{~J}$ and $8 \mathrm{~K}$ ), which may suggest that high level of PRICKLE2 is associated with poor prognosis. Moreover, oncomine analysis showed that PRICKLE2 expression was significantly upregulated in lymph node stages 3 of gastric cancer patients. Taken together, these results suggest that PRICKLE2 play an important role in the progression, invasion and migration of gastric cancer.

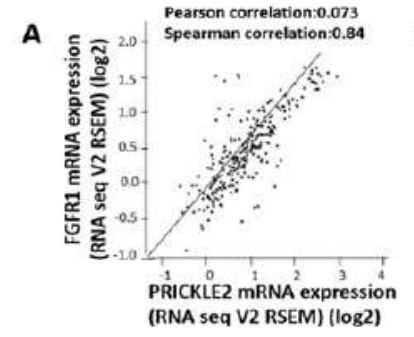

E

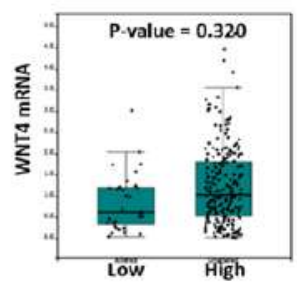

I

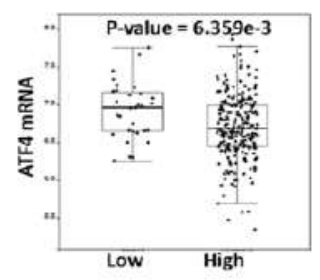

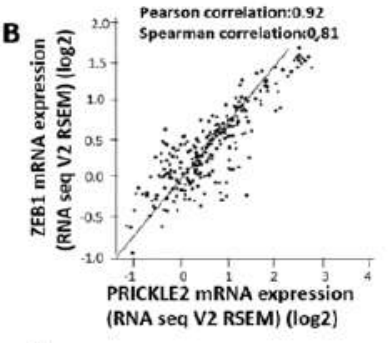

F

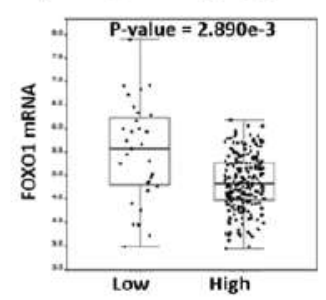

」

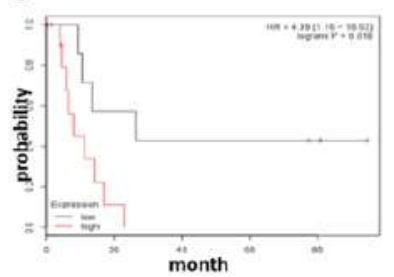

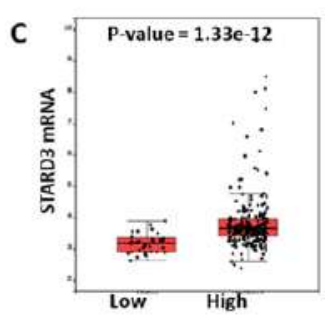

G
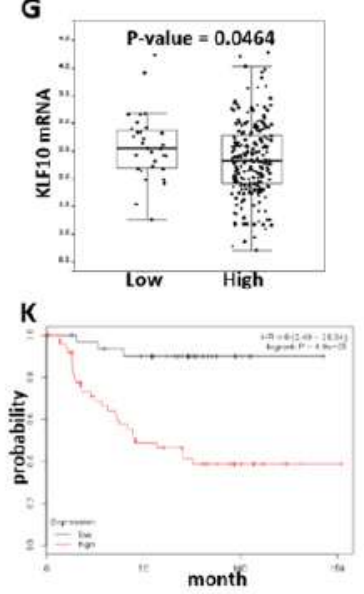
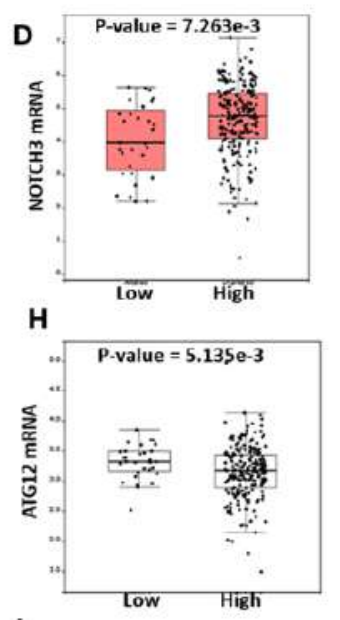

L Derico Gastric et al. PRICKLE2 Expression

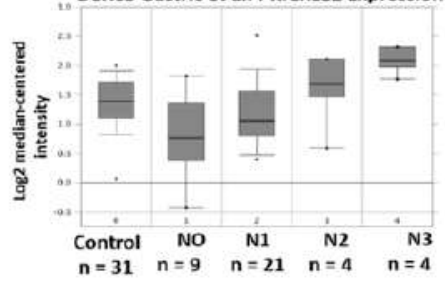

Figure 8. PRICKLE2 induces gastric cancer growth in GC samples

(A and B) Positive correlation of PRICKLE2 with FGFR1 and ZEB1. Analysis of GC samples data set fromcBioPortal cancer genomics database (http://www.cbioportal.org/index.do). Plots were drawn using Car package that contains a scatter plot function (http://cran.r-project.org). Linear regression analysis was used after log2transformation betweenPRICKLE2; and between PRICKLE2 and ZEB1.

(C-I) GC cancer dataset from (http://www.cbioportal.org/index.do) was divided into two groups having a high andlow expression of PRICKLE2.

(C-E) Analysis of STARD3, NOTCH3 and WNT4 expression showed PRICKLE2 enhances these genes expressions in GC samples. (F-I) Analysis of several tumor suppressorproteins showed PRICKLE2 inhibits tumor suppressor genes mRNA expressions in GC samples. Box plots showing changes in mRNA expression in GC samples having a high and low expression of DZIP. Box boundaries show $25^{\text {th }}$ and $75^{\text {th }}$ percentile; whisker shows $10^{\text {th }}$ and $90^{\text {th }}$ percentile; and the line represents the median value.

(J and K) Survival curves fromKM plotter (http://www.kmplot.com/) using GC samples showing that GC patients having levels of PRICKLE2 show poor survival. The GC samples were divided at the median value into two groups having a high and low expression of PRICKLE2.

(L) Oncomine database (http://www.oncomine.com) was used to analyze the PRICKLE2 expression in GC 18 19 samples dataset. Box plots showing upregulation of PRICKLE2 in GC patients' samples. Whisker shows $10_{\text {th }} 20$ and $90^{\text {th }}$ percentile; line represents the median value; box boundaries show $25^{\text {th }}$ and $75^{\text {th }}$ percentile. Range of data is represented by dots. 
Gastric cancer is among aggressive types of cancer, which metastasize very rapidly to secondary organs of the body. GC patients mainly develop local recurrence and distant metastasis. Amplification of oncogenic signaling play key role in GC progression, EMT, invasion and metastasis. However, underlying molecular mechanisms and identification of independent prognostic markers in GC remains poorly understood. In the present study, we aimed to identify independent and potential biomarkers for the diagnosis and better treatment of GC patients. Moreover, we also investigated novel molecular mechanisms involved in GC progression using GC samples. Our results provide new insights into GC progression and will be helpful in GC patient's clinical trials for effective treatment.

We found that SATB1, CLIP4, DZIP1 and PRICKLE2 serve as important prognostic markers in GC patient's samples. We show that SATB1, CLIP4, DZIP1 and PRICKLE2 expression enhance in GC samples and playkey role in GC progression. We also identified a subset of oncogenes that co-amplify with SATB1 in different subtypes of GC. Importantly, we observed a high percentage of SATB1 co-amplification with a subset of oncogenes in CIN and GS subtypes in comparison with MSI and EBV subtypes. These results suggest that SATB1 may play more aggressive role in CIN and GS subtypes of GC. Importantly, this finding also point towards that SATB1 may act as an independent prognosis marker in CIN and GS molecular subtypes of GC. CIN subtype of GC show amplification of growth factor receptor tyrosine kinases. One possible explanation of SATB1 amplification in CIN subtype is that growth factor receptor signaling may upregulate SATB1 expression to promote GC.

We observed positive correlation of SATB1 with a subset of oncogenes, which suggest that SATB1 may help other oncogenic proteins to further promote GC progression. More importantly, we found that SATB1 upregulates the mRNA levels of metastasis and EMT related genes in GC samples. These results suggest that SATB1 may play a key role in GC metastasis and EMT. Strikingly, we observed upregulation of WNT4, NOTCH3 and NOTCH4 expression in GC samples having high levels of SATB1. These finding suggest a novel link of SATB1 with WNT and NOTCH signaling pathway.

Importantly, we found that SATB1 down-regulates a panel of tumor suppressor genes in GC samples. These results further support the oncogenic role of SATB1 in GC progression. Induction of autophagy play very important role in the suppression of GC. Proteins such as ATF4, ATG12, BAG3 and PINK1 induce autophagy in cancer cells to suppress cancer progression. We found that expression of ATF4, ATG12, BAG3 and PINK1 was significantly downregulated in GC samples having high levels of SATB1. These results suggest that SATB1 may inhibit the autophagy process to promote GC progression. Strikingly, GC patients having high levels of SATB1 show poor survival rates. These results indicate that GC patients having SATB1 amplification develop more aggressive cancer and show worse survival.

The roles of CLIP4, DZIP1 and PRICKLE2 remain unknown in cancer progression. We identified for the first time that CLIP4, DZIP1 and PRICKLE2 genes involved in GC progression. Our results show that CLIP4, DZIP1 and PRICKLE2 enhance gastric cancer progression. CLIP4 show positive correlation with STARD8 and ZEB1 in GC samples, which suggest that CLIP4 may promote EMT of GC. DZIP1 enhance CXCR3/CXCR6 axis in GC samples, which indicate that DZIP1 promote tumorigenesis of GC. PRICKL2 promote the expression of WNT4 and NOTCH3 signaling proteins, which show that PRICKLE2 may induce GC progression by targeting WNT and NOTCH signaling pathway proteins. Moreover, we observed decrease expression of a subset of tumor suppressor genes in GC samples having high expressions of CLIP4, DZIP1 and PRICKLE2. GC patients having high levels of CLIP4, DZIP1 and PRICKLE2 show poor survival rates. Oncomine database results showed that CLIP4, DZIP1 and PRICKLE2 expressions are upregulated in the advance stages of GC. These results identified CLIP4, DZIP1 and PRICKLE2 as an independent and potential biomarker for the diagnosis of GC patients.

In summary, our results identified novel roles of SATB1, CLIP4, DZIP1 and PRICKLE2 in GC samples. SATB1, CLIP4, DZIP1 and PRICKLE2 play aggressive roles in GC progression. Importantly, SATB1, CLIP4, DZIP1 and PRICKLE2 enhance the expression of a subset of oncogenes in GC samples to further enhance GC progression. Expression of these proteins is negatively correlated with GC patient's survival. These resultsidentified SATB1, CLIP4, DZIP1 and PRICKLE2 as independent and potential biomarkers for better diagnosis and treatment of GC patients. These results also point towards the novel strategy by targeting SATB1, CLIP4, DZIP1 and PRICKLE2 in GC patients. Importantly, these results highlight for first time the role of CLIP4, DZIP1 and PRICKLE2 in cancer progression.

\section{Acknowledgment}

We are thankful of our lab colleagues for helpful suggestions and critically discussing our data.

\section{Competing financial interests}

The authors declare no competing financial interests.

\section{References}

1. Takahashi T, Saikawa Y, Kitagawa Y. Gastric cancer: current status of diagnosis and treatment. Cancers.2013;5(1):48-63. doi: 10.3390/cancers5010048.

2. Wadhwa R, Song S, Lee JS, Yao Y, Wei Q, Ajani JA. Gastric cancer molecular and clinical 
dimensions. Nat Rev ClinOncol. 2013;10(11):643 655. doi: $10.1038 /$ nrclinonc.2013.170.

3. Hu B, El Hajj N, Sittler S, Lammert N, Barnes R, Meloni-Ehrig A. Gastric cancer: classification, histologyand application of molecular pathology. J GastrointestOncol. 2012; 3(3):251-261.

4. Wong SS, Kim KM, Ting JC, Yu K, Fu J, Liu S, et al. Genomic landscape and genetic heterogeneity in gastric adenocarcinoma revealed by whole-genome sequencing. Nat Commun. 2014;5:5477.

doi: $10.1038 /$ ncomms6477.

5. Wang K, Yuen ST, Xu J, Lee SP, Yan HH, Shi ST, et al. Whole-genome sequencing and comprehensive molecular profiling identify new driver mutations in gastric cancer. Nat Genet. 2014;46(6):573-582. doi:10.1038/ng.2983.

6. Tan IB, Ivanova T, Lim KH, Ong CW, Deng N, Lee $\mathrm{J}$, et al. Intrinsic subtypes of gastric cancer, based on Gene expression pattern, predict survival and respond differently chemotherapy. Gastroenterology.2011; 141(2):476-485. doi: 10.1053/j.gastro.2011.04.042.

7. Lee YS, Cho YS, Lee GK, Lee S, Kim YW, Jho S, et al. Genomic profile analysis of diffuse-type gastric cancers. Genome Biol. 2014;15(4):R55. doi: 10.1186/ gb-2014-15-4-r55.

8. Cristescu R, Lee J, Nebozhyn M, Kim KM, Ting JC, Wong SS, et al. Molecular analysis of gastric cancer identifies subtypes associated with distinct clinical outcomes. Nat Med.2015;21(5):449-456. doi: $10.1038 / \mathrm{nm} .3850$.

9. Zang ZJ, Cutcutache I, Poon SL, Zhang SL, McPherson JR, Tao J, et al. Exome sequencing of gastric adenocarcinoma identifies recurrent somatic mutations in cell adhesion and chromatin remodeling genes. Nat Genet. 2012;44(5):570-574. doi: $10.1038 /$ ng.2246.

10. Shah MA, Khanin R, Tang L, Janjigian YY, Klimstra DS, Gerdes H, et al. Molecular classification of gastric cancer: a new paradigm. Clin Cancer Res. 2011;17(9):2693-2701. doi: 10.1158/1078-0432.CCR- 10-2203.

11. Li X, Wu WK, Xing R, Wong SH, Liu Y, Fang X, et al. Distinct subtypes of gastric cancer defined by molecular characterization include novel mutational signatures with prognostic capability. CancerRes.2016; 76(7):1724-1732. doi: 10.1158/00085472.CAN-15-2443.

12. Ooi CH, Ivanova T, Wu J, Lee M, Tan IB, Tao J, et al. Oncogenic pathway combinations predict clinical prognosis in gastric cancer. PLoS Genet. 2009; 5(10):e1000676. doi: 10.1371/journal.pgen.1000676.

13. Han HJ, Russo J, KohwiY ,KohwiShigematsu T. SATB1 reprograms gene expression to promote breast tumor growth and metastasis. Nature. 2008;452(7184):187-193.

14. Cheng $\mathrm{C}, \mathrm{Lu} \mathrm{X}$, Wang $\mathrm{G}$, et al. Expression of SATB1 and heparanase in gastric cancer and its relationship to clinicopathologic features. APMIS. 2010;118(11): 855-863.

15. Lu X, Cheng $\mathrm{C}$, Zhu S, et al. SATB1 is an independent prognostic marker for gastric cancer in a Chinese population. Oncol Rep. 2010;24(4):981987.

16. Zhang J, Zhang B, Zhang X, et al. SATB1 expression is associated with biologic behavior in colorectal carcinoma in vitro and in vivo. PLoS One. 2013;8(1):e47902.

17. Tu W, Luo M, Wang $\mathrm{Z}$, et al. Upregulation of SATB1 promotes tumor growth and metastasis in liver cancer. Liver Int. 2012;32(7):1064-1078.

18. Xiang J, Zhou L, Li S, et al. AT-rich sequence binding protein 1: contribution to tumor progression and metastasis of human ovarian carcinoma. Oncol Lett. 2012;3(4):865-870.

19. Lv JH, et al. SATB1 expression is correlated with $\beta$ catenin associated epithelial-mesenchymal transition in colorectal cancer. Cancer BiolTher. 2016 Mar 3; 17(3):254-61. doi: 10.1080/15384047.2016.1139239. Epub7 2016 Jan 25.

20. Mansour MA et al; SATB1 and SATB2 play opposing roles in c-Mycexpression andprogression of cancer. Oncotarget. 2016 Jan 26;7(4):4993-5006. doi: 10.18632/ oncotarget.6651.

21. Lv C, et al; MicroRNA-495 suppresses human renal cell carcinoma malignancy by targeting SATB1. Am J Transl Res. 2015 Oct 15;7(10):1992-9. E Collection 2015.

22. Wang G; miR-23a suppresses proliferation of osteosarcoma cells by targeting SATB1. Tumor Biol. 2015Jun;36(6):4715-21. doi: 10.1007/s13277-0153120-0. Epub 2015 Jan 27.

23. Wang F, et al; Positive feedback between RNAbinding protein $\mathrm{HuD}$ and transcriptionfactor SATB1 promotes neurogenesis. Proc Natl AcadSci U S A 2015 Sep8;112(36):E4995-5004. doi:10.1073/pnas. 1513780112. Epub 2015 Aug 24.

24. Yang Y, et; SATB1 Mediates Long-Range Chromatin Interactions: A Dual Regulator of AntiApoptotic BCL2 and Pro-Apoptotic NOXA Genes. PLoS One. 2015 Sep 30;10(9):e0139170. doi: 10.1371/journal. pone.0139170. e Collection 2015.

25. Nodin B, Hedner C, Uhlen M, Jirström K. Expression of the global regulator SATB1 is an independent factor of poor prognosis in high grade epithelial ovarian cancer. J Ovarian Res. 2012;5(1):2.

26. Shukla S, Sharma H, Abbas A, et al. Upregulation of SATB1 is associated with prostate cancer aggressiveness and disease progression. PLoS One. 2013;8(1):e53527.

27. Mao L, Yang $\mathrm{C}$, Wang $\mathrm{J}$, et al. SATB1 is overexpressed in metastatic prostate cancer and promotes prostate cancer cell growth and invasion. $\mathrm{J}$ Transl Med. 2013;11: 111. 
28. Han B, Luan L, Xu Z, Wu B. Expression and biological roles of SATB1 in human bladder cancer. Tumor Biol. 2013;34(5):2943-2949.

29. Ota $\mathrm{T}$, et al. Complete sequencing and characterization of 21,243 full-length human cDNAs. Nat Genet, 2004 Jan. PMID 14702039 Rolland T, et al; A proteome-scale map of the human interactome network.. Cell, 2014 Nov20. PMID 25416956.

30. Kimura K, Wakamatsu A, Suzuki Y, et al. (2006). "Diversification of transcriptional modulation: largescale identification and characterization of putative alternative promoters of human genes.". Genome Res. 16(1):65.doi:10.1101/gr.4039406.PMC1356129. PMID 16344560.

31. Kimura K, Wakamatsu A, Suzuki Y, et al. (2006)."Diversification of transcriptional modulation: large-scale identification and characterization of putative alternative promoters of human genes.". Genome Res. 16 (1): 5565.doi:10.1101/gr.4039406. PMC 1356129.PMID 16344560 .

32. Zhang B, et al; GSK3 $\beta$-Dzip1-Rab8 cascade regulates ciliogenesis after mitosis. PLoS Biol. 2015 Apr10;13(4):e1002129. doi: 10.1371/journal.pbio. 1002129. e Collection 2015 Apr.
33. Mei X, et al; Functional characterization of Prickle2 and BBS7 identify overlapping phenotypes yet distinct mechanisms. Dev Biol. 2014 Aug 15;392(2): 245-55. doi: 10.1016/j.ydbio.2014.05.020. Epub 2014 Jun 2.

34. Cancer Genome Atlas Research Network. Comprehensive molecular characterization of gastric adenocarcinoma. Nature. 2014 Sep 11;513(7517): 202-9. doi: 10.1038/nature13480. Epub 2014 Jul 23.

35. Liu H, et al; Long non-coding RNAs as prognostic markers in human breast cancer. Oncotarget. 2016 Mar 1. doi: 10.18632/oncotarget.7828.

36. Klonowska $\mathrm{K}$, et al; Oncogenomic portals for the visualization and analysis of genome-wide cancer data.Oncotarget.

37. Chen WC, et al; Systematic Analysis of Gene Expression Alterations and Clinical Outcomes for Long-Chain Acyl Coenzyme A Synthetase Family in Cancer. PLoS One 2016 May 12;11(5): e 0155660. doi:10.1371/journal.pone.0155660.

38. Charina E. Ortega, Yoshua Seidner, and Isabel Dominguez. "Mining CK2 in Cancer. PLoS One. 2014;9(12):e115609. Published online 2014 Dec26. doi: $\quad$ 10.1371/journal.pone.0115609 